\title{
Registration Parameter Spaces for Molecular Electron Tomography Images
}

\author{
Lennart Svensson`, Anders Brun, Ingela Nyström, and Ida-Maria Sintorn \\ Centre for Image Analysis, \\ Swedish University of Agricultural Sciences and \\ Uppsala University, Sweden \\ \{lennart, anders, ingela, ida\}@cb.uu.se \\ http://www.cb.uu.se
}

\begin{abstract}
We describe a methodology for exploring the parameter spaces of rigid-body registrations in 3-D. It serves as a tool for guiding and assisting a user in an interactive registration process. An exhaustive search is performed over all positions and rotations of the template, resulting in a 6 -D volume, or fitness landscape. This is explored by the user, who selects and views suitable 3 -D projections of the data, visualized using volume rendering. The 3 -D projections demonstrated here are the maximum and average intensity projections of the rotation parameters and a projection of the rotation parameter for fixed translation parameters. This allows the user to jointly visualize projections of the parameter space, the local behaviour of the similarity score, and the corresponding registration of the two volumes in 3-D space for a chosen point in the parameter space. The procedure is intended to be used with haptic exploration and interaction. We demonstrate the methodology on a synthetic test case and on real molecular electron tomography data using normalized cross correlation as similarity score.
\end{abstract}

Keywords: volumetric registration, template matching, normalized cross correlation, molecular electron tomography.

\section{Introduction}

Optimization in high dimensional spaces is a difficult problem for any complex function. For simpler functions, e.g. monotonically increasing, exploitation schemes based on gradients can yield good results, but when more emphasis needs to be put on exploration due to local optima, the process can become too slow or the methods still easily get stuck in local optima. Both rigid and non-rigid registration are problems where the search space often is high dimensional. Here we describe a methodology for how rigid body registration parameter spaces can be presented and investigated to let the user guide the optimization process and simultaneously analyze the registration result.

^ Corresponding author.

G. Maino and G.L. Foresti (Eds.): ICIAP 2011, Part I, LNCS 6978, pp. 403-412, 2011.

(C) Springer-Verlag Berlin Heidelberg 2011 
The application in this paper concerns Molecular Electron Tomography (MET) data, which is cryo electron microscopy combined with 3 -D reconstruction techniques to create detailed tomograms of biological samples at the nanometer scale. MET allows for studying the structure, flexibility and interactions of molecules and macromolecules in solution (in vitro) as well as in tissue samples (in situ). A characteristic feature of MET volumes is however a very low signal to noise ratio. Registration is a very important process when analyzing this kind of data as it is very difficult to visually identify the objects or regions of interest in the very cluttered and complex volume images, and it is also difficult to analyze interactions and relations between different objects only based on vision.

Registration is usually performed with crystallographic data. The software Situs published in 1999 [12 has become a popular tool for this. It originally featured rigid body registration based on cross correlation. Shortly after, non-rigid registration was introduced by, e.g., Wriggers [10, who used a vector quantization registration scheme using restraints between vectors as a model for the structure flexibility. Birmanns introduced the use of haptics [2] to explore this landscape interactively and our approach is along the same lines.

The fitness landscape of rigid registration is defined by a scalar similarity function defined in the 6-D parameter space. We introduce projections of the fitness landscape into 3-D score volumes, visualized using a volume renderer, which allows the user to explore and navigate this high-dimensional space in search for relevant matches. This process is explained in Figure 1. It allows the user to simultaneously explore both the ordinary 3-D spatial domain, in which the registration is performed, and projections of the 6-D parameter space of rigid registration. In our application domain, the visualization and analysis of MET biological data, this methodology will be used in conjunction with haptic rendering and interaction, where a "3-D pen" with force feedback will be used to position and rotate the template image in the search image.

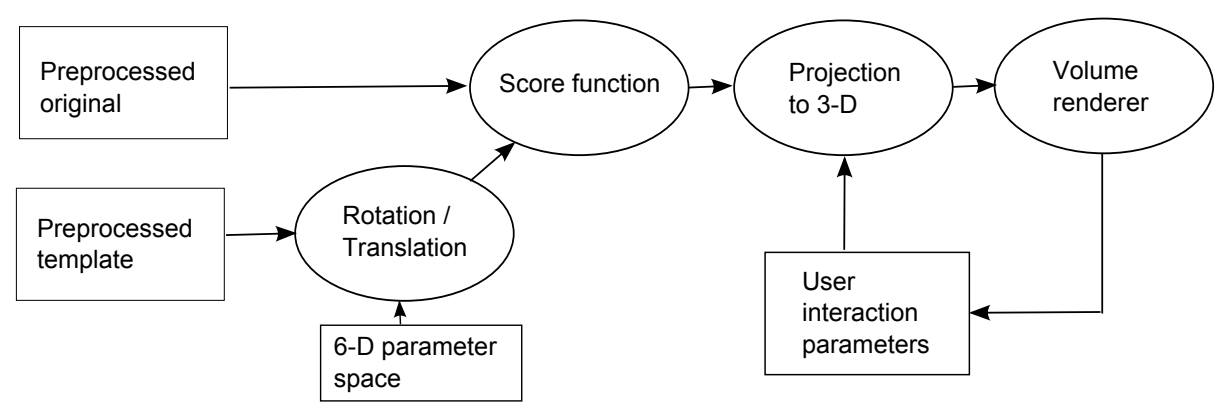

Fig. 1. Flowchart for the methodology 


\section{Methodology}

Rigid-body registration involves six degrees of freedom: three degrees for translation, $\mathbf{t} \in \mathbf{R}^{3}$, and three for rotation, $\mathbf{A} \in S O(3)$. The special orthogonal group $S O(3)$ is the set of all rotation matrices.

To compute the total score volume, $s: \mathbb{R}^{3} \times S O(3) \rightarrow \mathbb{R}$, we start by preprocessing the image data. Voxel values below a user set level, corresponding to the background noise level, are removed. An exhaustive registration search is then performed. We match the template with the original image volume using rotations and translations of the template evenly distributed in the image volume (see Sections 2.1 and 2.2).

The similarity function $s$, defined in the total parameter space, cannot be visualized in one 3 -D view. For this reason, some kind of dimensionality reduction is required. We have taken two approaches in this paper to collapse this function which both provide $3-\mathrm{D}$ volumetric projections suitable for exploring the parameter space of rigid registration:

- Mapping $s: \mathbb{R}^{3} \times S O(3) \rightarrow \mathbb{R}$ to $f: \mathbb{R}^{3} \rightarrow \mathbb{R}$, by summarizing the information over the rotation subspace (see Section 2.3 ). The result is a rectangular $3-\mathrm{D}$ score volume, with the same dimensions as the original image, which provides an overview of the fitness landscape.

- Mapping $s: \mathbb{R}^{3} \times S O(3) \rightarrow \mathbb{R}$ to $g: S O(3) \rightarrow \mathbb{R}$, by keeping a particular translation fixed (see Section 2.4). The result is a scalar function defined in a ball in 3-D, which provides a view of the fitness landscape of all rotations when a particular translation is fixed.

\subsection{Distribution of Rotation Angles}

Uniform sampling of the set of all rotations, $S O(3)$, is a non-trivial problem, which has been addressed in many publications, see e.g., 13. Random sampling of $S O(3)$ is easier to facilitate, since rotations may be described by the set of unit quaternions (with antipodal points identified). Thus, random sampling of $S O(3)$ is equivalent to random sampling of the unit sphere in $\mathbb{R}^{4}$. However, random sampling introduces noise in the sampling, which makes the representation inefficient. To fix this, a relaxation procedure was devised to make the sampling more uniform.

1. Randomly sample $N$ points on a unit 3 -sphere in $\mathbb{R}^{4}$, store as rows in a $N \times 4$ matrix $\tilde{\mathbf{X}}^{(0)}$.

2. Double the dataset by mirroring, $\mathbf{X}^{(0)}=\left[\tilde{\mathbf{X}}^{(0)} ;-\tilde{\mathbf{X}}^{(0)}\right]$.

3. Compute all pairwise Euclidean distances, store in a $2 N \times 2 N$ matrix $\mathbf{D}$.

4. Compute a weighted Laplacian matrix, $\mathbf{W}$, with coefficients: $W_{i j}=$ $-1 /\left(D_{i j}+1\right)^{\alpha}$, when $i \neq j$, and $W_{i i}=-\sum_{k \neq i} W_{i k}$.

5. Relax points $\mathbf{X}^{(m+1)}=(\mathbf{I}+\mathbf{W}) \mathbf{X}^{(m)}$.

6. Normalize each row in $\mathbf{X}$ to unit length.

7. If not converged, go to 3 .

8. Remove the mirrored half of the points. 
Running this scheme, it was observed that the average distance from a point to its closest neighbor increased with around $80 \%$, for $N=1000, \alpha=20$ after 50 relaxation iterations. This indicates that the sampling becomes more uniform and thus also more efficient. By the use of a mirrored set of points, we ensured that the sampling was uniformly sampled from the set of antipodally identified points on the sphere in $\mathbb{R}^{4}$.

\section{$2.2 \quad$ Similarity Measure}

Choosing an appropriate scoring function is to a large extent a trade-off between accuracy and implementation speed. A thorough investigation of this subject was done by Wriggers [1]. Mutual information is an alternative similarity measure that was not covered by Wriggers. It is an established scoring function for many (medical) imaging modalities and has been found to work well also for this kind of data 9. However, the extra generality of this measure, such as handling non-linear differences, was not needed in this case. Here, normalized cross-correlation [3] was chosen:

$$
s(\mathbf{u})=\frac{\sum_{\mathbf{x}}\left(I(\mathbf{x})-\bar{I}_{\mathbf{u}}\right)(T(\mathbf{x}-\mathbf{u})-\bar{T})}{\left(\sum_{\mathbf{x}}\left(I(\mathbf{x})-\bar{I}_{\mathbf{u}}\right)^{2} \sum_{\mathbf{x}}(T(\mathbf{x}-\mathbf{u})-\bar{T})^{2}\right)^{1 / 2}}
$$

where $\bar{T}$ is the mean of the template and $\bar{I}_{\mathbf{u}}$ is the mean of the region under the template. The computation of normalized correlation was performed in the Fourier domain for computational efficiency [5].

\subsection{Summarizing over the Rotation Subspace}

We use the $p$-norm to summarize a scalar function over $S O(3)$. For any translation $\mathbf{t} \in \mathbb{R}^{3}$, we define

$$
f(\mathbf{t})=\left(\sum_{\mathbf{A} \in S O(3)} s(\mathbf{t}, \mathbf{A})^{p}\right)^{1 / p}
$$

where $p \in[1, \infty[$. Cases of particular interest are:

- maximum projection, $p=\infty$, and

- average projection, $p=1$.

To obtain a strong response with the average projection, the object needs to be rotationally symmetric, which is quite often the case for biological molecules. The combined analysis of the average and maximum projections is useful for 
identifying good registration positions for symmetric objects. They will have strong responses at good registration positions in both projections. It is similarly useful for discarding false positions for non symmetrical objects since, for good/correct positions, they will only have a strong response in the maximum projection. An alternative to simultaneously analyzing two projection volumes is to look at one projection using a different $p$-norm, which corresponds to mixing the maximum and average projection.

For analyzing and interpreting the score volumes the position of the center point of the template need to be considered. For symmetrical objects to generate strong and focused responses in the score volumes the rotation center of the template needs to be positioned midway on the symmetry axis/axes. If not, it will lead to multiple or smeared out and less pronounced maxima around the correct positions in the score volumes. If, for example, an object is relatively rotationally symmetric around one axis and the rotation center is positioned on that symmetry axis but not midway it will give rise to double maxima in the translation score volumes. Picture a vertical stick with the center point on the stick but somewhat offset from half of the length of the stick. One maximum will then correspond to the "correct" match and one to the upside down match.

\subsection{Fixing the Translation in $\mathbb{R}^{3}$}

For a particular translation $\mathbf{t} \in \mathbb{R}^{3}$, selected by the user, we define

$$
g(\mathbf{A})=s(\mathbf{t}, \mathbf{A})
$$

Via Euler's theorem, $g(\mathbf{A})$ may then be visualized as a scalar function defined in a ball with radius $\pi$ in $\mathbb{R}^{3}$. In this ball, the identity rotation is placed at the center. Points within the ball specify an additional rotation around the vector from the center to the point itself, where the euclidean norm of the vector specifies the rotation angle.

Euler's theorem states that any rotation in 3-D can be described by a rotation around a particular axis, i.e., parameterized by the set of unit vectors, $\|\hat{\mathbf{n}}\|=1$, and a rotation angle, $\alpha \in(0, \pi)$. The mapping $h: S O(3) \rightarrow \mathbb{R}^{3}, \mathbf{y}=h(\hat{\mathbf{n}}, \alpha)=$ $\alpha \hat{\mathbf{n}}$ provides a convenient coordinate system for visualizing the set of all rotations as a volume. In this mapping, every rotation is mapped to a point inside a ball with radius $\pi$. In order to place the maximum of $g(\mathbf{A})$ in the center of the sphere, $\hat{g}(\mathbf{A})=g\left(\mathbf{A} \mathbf{A}_{\max }^{-1}\right)$ is visualized where $\mathbf{A}_{\max }$ is the rotation with the maximum value of $g(\mathbf{A})$.

\section{Experiments}

The proposed methodology is illustrated on three different types of volumes and templates: a synthetic volume, a MET volume of antibodies in solution, and a MET volume of in situ skin cells. For each image type the original volume, translation score volumes, a rotation score volume, and registrations corresponding to different optima in the parameter spaces are shown. 


\subsection{Synthetic Data Set}

For a first assessment, a synthetic volume image was generated, which is shown in Figure 2 (a). It consists of one structure with three perpendicular arms of different lengths, and is not intended to be a replica of real data, but a suitable test structure to highlight features of the methodology. It is not symmetrical around any axis and the inner parts of the structure have higher intensity. In the search, we used a spatially identical template with linearly mapped intensities.

Figure 2(b)-(d) show three different translation score volumes corresponding to $p=1$ (average projection), $p=8$, and $p=\infty$ (maximum projection). For this artificial example with flat background and only one object all three projections clearly show a maximum for the correct translation parameters and lower scores (intensities) for positions when the template partly overlaps the object. Figure 2 (e) shows the rotation projection for translation parameters interactively picked from the maximum in the $p=\infty$ translation projection. The center in that particular volume has the highest matching value and corresponds to the correct registration shown in Figure2 (f). The two second highest optima in Figure 2(e), correspond to the two rotations of the template where the directions of all the arms coincide with the directions of the object arms. The registration corresponding to one such rotation is shown in Figure 2 (g). The six slightly lower optima (two on vertical "line" and four "dots") correspond to rotations of the template where only two of the arms overlap with the object arms. One such registration is shown in Figure 2 (h).

\subsection{IgG Data Set}

The second test case, is illustrated in Figure 3. It consists of a part of a volume of proteins (antibodies) in solution, where a single Immunoglobulin G, IgG, antibody (seen in the center of Figure 3 (a)), has been identified [8]. The IgG molecule has three subgroups connected at one joint. In the test volume, the three parts and the center joint are roughly spanning a plane. The antibody template was created from the protein's atom positions deposited in the protein data bank (PDB) [164]. A volume image where intensity represents density is constructed by placing a Gauss-kernel, with standard deviation $1 \mathrm{~nm}$, at each atom position weighted by the atom mass, giving a resolution of $2 \mathrm{~nm}$. The intensity in each voxel in the image is generated by adding contributions from all Gauss-kernels in the vicinity of that voxel [7].

Figure 3 (b)-(d) show the translation score volumes for, $p=1$ (average projection), $p=8$, and $p=\infty$ (maximum projection). For this image with lots of small objects the benefit of studying different projections is especially large. The correct position is not a pronounced maximum in the average projection but it is in the $p=8$ and maximum projections. In fact it is the only pronounced optimum in the maximum projection score volume.

Figure 3 (e) shows the rotation score volume for a set of translational parameters interactively picked from the maximum in Figure 3 (d). In the rotational subspace for the best translational position, Figure 3 (e), different local maxima 


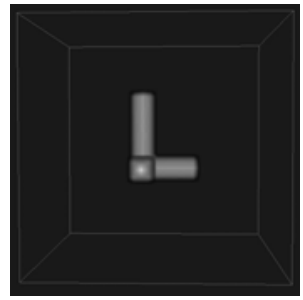

(a) Test volume



(b) Score vol, $p=1$

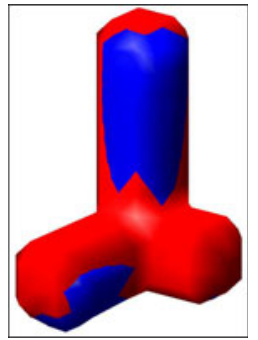

(f) Best reg.

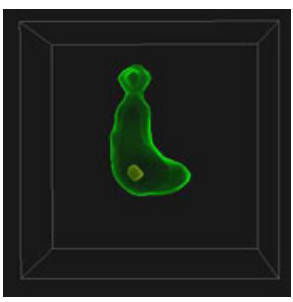

(c) $p=8$



(g) Local max

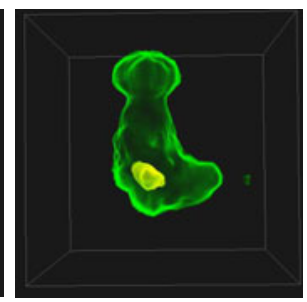

(d) $p=\infty$

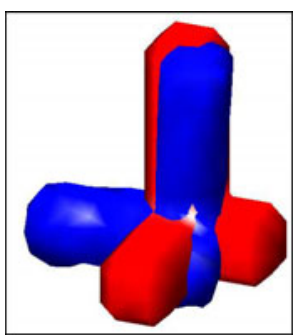

(h) Local max

(e) Rotational score vol.

Fig. 2. The synthetic test volume (a), translation score volumes (b)-(d), rotation score volume (e), and different registration results with the image volume structure in red and search template in blue (f)-(h). The score volumes have been rendered using an isosurface for low scores seen in low intensity, and a higher intensity isosurface for higher scores.

are visible that correspond to different orientations yielding a fairly good match. The arms and stem of the IgG are similar so if they are matched to each other in the wrong way, a high score is still acquired. Antipodal points (on the opposite side of the ball surface) refer to the same orientation. For a tri-symmetrical planar object, a total of eleven pronounced local maxima (central maximum plus five $\times 2$ antipodal maxima) would be seen in the rotation score volume. In this case with IgG which is relatively symmetrical and relatively planar only nine pronounced local maxima (central maximum plus four $\times$ two antipodal maxima) are seen with the isosurface rendering levels chosen in Figure 3 (e).

One maxima is connected to two other maxima through a path of slightly higher intensities in the rotation score volume. Although not visible in this rendering such connecting paths can be seen for all the maxima. These paths of slightly higher match scores correspond to rotating the template while one component is fixed and overlapping one of the object components. Figure 3 (f) shows the registration corresponding to the center maximum in (e) and Fig. 3 (g) shows the registration corresponding to one of the other matches seen as non-central local maxima in (e). 


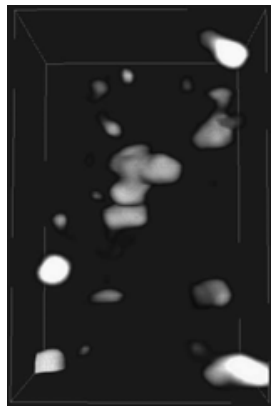

(a) Test volume

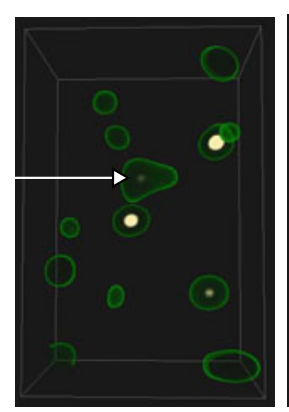

(b) Score vol, $p=1$

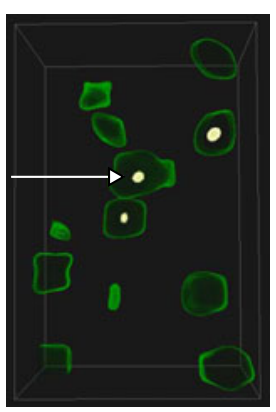

(c) $p=8$

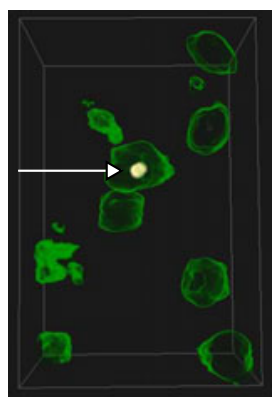

(d) $p=\infty$

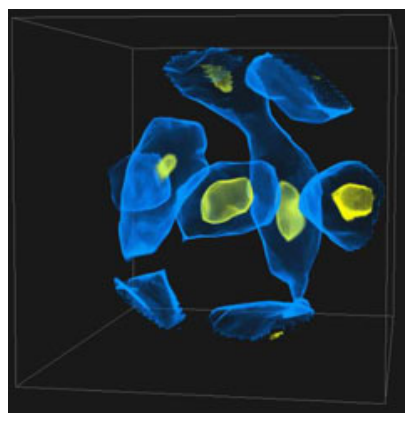

(e) Rot. score vol.



(f) Best reg.

(g) Local max

Fig. 3. The IgG test volume (a), translation score volumes with (b) - (d), rotation score volume (e), and different registration results (f) $-(\mathrm{g})$. Since the $\mathrm{IgG}$ is rotationally asymmetric, the average projection yields a weak response for the correct position (marked with arrow), but as $\mathrm{p}$ is increased towards maximum projection, the correct position becomes clearer.

\subsection{Desmosome Data Set}

The third investigated data set, which can be seen in Figure 4, concerns a MET volume from a skin sample. The structures of interest in these volumes are chains of two corneodesmosin antibodies linked to a very bright and spherical gold particle. In Figure 4 (a) four such chains are visible as bright and slightly elongated structures. The template used is one of the chains cut from the volume and the task is to identify the three other chains.

The antibody chains in this volume are elongated structures, which are relatively rotationally symmetric around one axis. This implies mainly two things when interpreting the score volumes. Firstly that significant responses for the correct positions in average translation score volume would be expected. Secondly that, due to imperfectly positioned rotation point, the local maxima might be rather blurry in the average projection and multiple local maxima might be found for the correct position in all score volumes but especially in the maximum projection score volume. These phenomena can indeed be seen in the score 


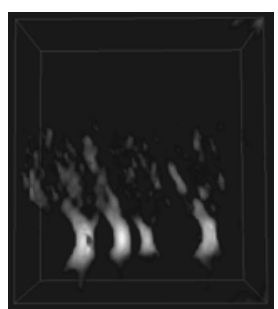

(a) Test volume

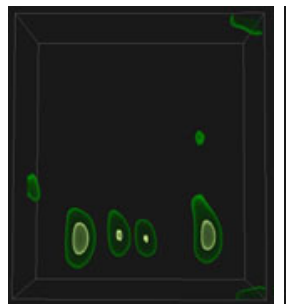

(b) $p=1$

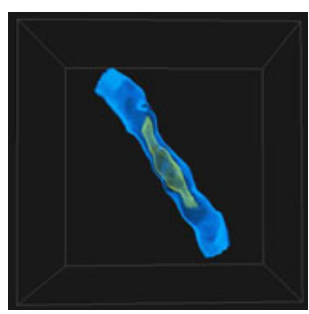

(e) Rot. score volume



(c) $p=8$

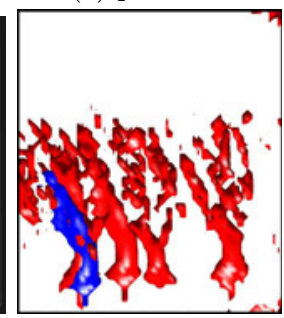

(f) Local max



(d) $p=\infty$

Fig. 4. The desmosome test volume (a). Rotation score volume for a correct translation position (b). Translation score volumes (c) - (e) One registration result (f).

volumes. For the maximum projection case, Figure 4 (d), double maxima can be found for each antibody chain, one correct above the gold particle and one below corresponding to the "upside down" match. The average projection, Figure 4(b), gives a strong but rather blurred response, centered at the gold particle, which is a displacement to the correct position. The rotation score volume for the correct translation position for one of the three antibody chains searched for has a clear cylinder shape, corresponding to rotations around the chain axis.

\section{Conclusion and Future Work}

We have presented a methodology for semi-automatic registration of MET data. The maximum projection has proven to be the overall best technique to summarize the non-spatial dimensions in the parameter space. The exploration of the rotational subspace, keeping the translation fixed, has also proven to be a valuable tool to understand the nature of a particular local maximum of the score function in the 6-D parameter space. In particular it reveals the maxima caused by symmetries, which are common in biological molecules.

In future work, we will include haptic rendering to give the user even stronger cues on the local fitting of the template volume. The projection from $6-\mathrm{D}$ to $3-\mathrm{D}$, through $p$-norms, looks different depending on the choice of this center. Different ways of automatically determining this center is a possible future topic. We also aim to explore more ways to summarize the 6-D parameter space. In particular, the $p$-norms used in this paper does not take the shape of the local maxima and 
the local fitness landscape into account when it summarizes the score function over all rotations.

Acknowledgment. This work is part of the ProViz project 11 and funded through the Visualization Program by the Knowledge Foundation, Vårdal Foundation, the Foundation for Strategic Research, VINNOVA, and Invest in Sweden Agency.

Thanks to: Stina Svensson, Sara Sandin, Aurelie Laloef, Lars Norlén, and Daniel Evestedt, for project management, providing images, and assisting in implementation of the pre-integrated ray-caster used.

\section{References}

1. Berman, H., Westbrook, J., Feng, Z., Gilliland, G., Bhat, T., Weissig, H., Shindyalova, I., Bourne, P.: The protein data bank. Nucleic Acids Research 28, 235-242 (2000)

2. Birmanns, S., Wriggers, W.: Interactive fitting augmented by force-feedback and virtual reality. Journal of Structural Biology 144(1-2), 123-131 (2003)

3. Gonzalez, R.C., Woods, R.E.: Digital Image Processing, 3rd edn., vol. ch. 12. Prentice-Hall, Inc., Upper Saddle River (2006)

4. Harris, L.J., Larson, S.B., Hasel, K.W., McPherson, A.: Refined structure of an intact IgG2a monoclonal antibody. Biochemistry 36(7), 1581-1597 (1997)

5. Lewis, J.P.: Fast normalized cross-correlation (1995)

6. http://www.pdg.org/ (visited December 7, 2010)

7. Pittet, J.J., Henn, C., Engel, A., Heymann, J.B.: Visualizing 3D data obtained from microscopy on the internet. Journal of Structural Biology 125, 123-132 (1999)

8. Sandin, S., Öfverstedt, L.G., Wikström, A.C., Wrange, O., Skoglund, U.: Structure and flexibility of individual immunoglobulin G molecules in solution. Structure 12, 409-415 (2004)

9. Telenczuk, B., Ledesma-Carbato, M., Velazquez-Muriel, J., Sorzano, C., Carazo, J.M., Santos, A.: Molecular image registration using mutual information and differential evolution optimization. In: 3rd IEEE International Symposium on Biomedical Imaging: Nano to Macro, pp. 844-847 (2006)

10. Wriggers, W., Birmanns, S.: Using situs for flexible and rigid-body fitting of multiresolution single-molecule data. Journal of Structural Biology 133(2-3), 193-202 (2001)

11. Wriggers, W., Chacon, P.: Modeling tricks and fitting techniques for multiresolution structures. Structure 9, 779-788 (2001)

12. Wriggers, W., Milligan, R.A., McCammon, J.A.: Situs: A package for docking crystal structures into low-resolution maps from electron microscopy. Journal of Structural Biology 125(2-3), 185-195 (1999)

13. Yershova, A., Jain, S., Lavalle, S.M., Mitchell, J.C.: Generating uniform incremental grids on $\mathrm{SO}(3)$ using the Hopf fibration. Int. J. Rob. Res. 29, 801-812 (2010)

$\overline{{ }^{1} \text { http://www.cb.uu.se/research/proviz/ }}$ 